\title{
UNSTABLE GROUND STATE OF NONLINEAR KLEIN-GORDON EQUATIONS
}

\author{
BY
}

JALAL SHATAH

\begin{abstract}
In this paper we prove the instability of the ground state, i.e. least energy steady-state solution of nonlinear Klein-Gordon equations with space dimension $n \geqslant 3$.
\end{abstract}

0. Introduction. We present here a proof of instability of the "ground state", the least energy steady-state solution of the equation

$$
u_{t t}-\Delta u+f(|u|) \arg u=0, \quad x \in \mathbf{R}^{n}, n \geqslant 3 .
$$

The instability of the ground state is shown for very general nonlinearities $f$. The only assumptions on $f$ are those that insure the existence of a nontrivial steady state solution of (0.1). If we denote the ground state by $u_{e}$, then we have

THEOREM. Let $u(t)$ be a solution of $(0.1)$ such that $u(0)=u_{0}, u_{t}(0)=u_{1}$. Then there are initial data $\left(u_{0}, u_{1}\right)$ arbitrarily close to $\left(u_{e}, 0\right)$ and a sequence $\left(t_{k}\right)$ such that $\left\|u\left(t_{k}\right)\right\| \rightarrow \infty$ as $k \rightarrow \infty$.

The theory of linearized stability gives a clue to the instability of the ground state since the linearized equation $v_{t t}-\Delta v+f^{\prime}\left(u_{e}\right) v=0$ has a negative eigenvalue.

The stability question was first considered by Derrick [3] who formally used the linearized stability argument to show that the ground state is unstable. There are several results on blow up for special nonlinearities such as Payne and Sattinger [6] and Berestycki and Cazenave [2]. However for certain nonlinearities the solutions do not blow up in finite time but exist globally in time. Therefore to solve this problem we are forced to introduce a new estimate that shows the instability of the ground state without blow up for all types of nonlinearities.

The existence of the ground state is basically due to Walter Strauss [10]. However in the most general setting (and this is the case we are dealing with here) this result is due to $\mathrm{H}$. Berestycki and P. Lions [12].

The method we use for obtaining the ground state is that of Clayton Keller who studied the flow of the equation $u_{t t}+u_{t}-\Delta u+f(u)=0$ near a stationary state. Keller showed that the stationary state is a saddle point with an infinite dimensional stable manifold and a nonempty finite dimensional unstable manifold.

Finally it is interesting to compare this result with the "degenerate" one-dimensional case. In this case it is well known that there are stable stationary solutions and even solitons, as in the case of the equation $u_{t t}-u_{x x}+\sin u=0$.

Received by the editors August 29, 1983 and, in revised form, November 19, 1984.

1980 Mathematics Subject Classification. Primary 35L05; Secondary 35J05. 
I would like to thank Professor Walter Strauss for his helpful remarks.

Notation. We employ here the standard notations

$$
\begin{gathered}
H_{r}^{1}\left(\mathbf{R}^{n}\right)=\left\{u, \text { radially symmetric functions on } \mathbf{R}^{n},\right. \\
\left.\|u\| \equiv\left(\int|\nabla u(x)|^{2} d x+\int|u(x)|^{2} d x\right)^{1 / 2}<\infty\right\}, \\
L_{r}^{p}\left(\mathbf{R}^{n}\right)=\left\{u, \text { radially symmetric functions on } \mathbf{R}^{n},\right. \\
\left.|u|_{p}=\left(\int|u(x)|^{p} d x\right)^{1 / p}<\infty\right\} .
\end{gathered}
$$

$u_{r}=\partial u / \partial r$ for $u \in H_{r}^{1}\left(\mathbf{R}^{n}\right)$, where $r=|x|, x \in \mathbf{R}^{n}$ and $\arg u=u /|u|$.

1. Existence of a nontrivial stationary solution. In this section we show the existence of a nontrivial solution to the problem

$$
-\Delta u+f(|u|) \arg u=0, \quad x \in \mathbf{R}^{n}, \quad n \geqslant 3,
$$

where $f(|u|)$ satisfies the following assumptions:

(i) $f(0)=0$ and $f^{\prime}(0)=1$.

(ii) $\lim f(|u|) /|u|^{l} \geqslant 0$ as $|u| \rightarrow \infty, l=1+4 /(n-2)$.

(iii) There is a $u$ such that $G(|u|)<0$, where $G^{\prime}(|u|)=f(|u|)$ and $G(0)=0$.

In this generality this problem was solved by $\mathrm{H}$. Berestycki and P. Lions [12]. For the purpose of showing instability we present here a proof by a method of C. Keller [5]. First we set up a minimization problem of a functional $J(u)$ restricted to a manifold $M$ and then show that this minimum is a solution of (1.1).

DEFINITION 1.1.

$$
\begin{aligned}
& J(u)=\frac{1}{2} \int|\nabla u|^{2} d x+\int G(|u|) d x, \\
& K(u)=\frac{n-2}{2} \int|\nabla u|^{2} d x+n \int G(|u|) d x, \\
& M=\left\{u \in H_{r}^{1}: K(u)=0, u \neq 0\right\} .
\end{aligned}
$$

The space $H_{r}^{1}\left(\mathbf{R}^{n}\right)$ has the following property.

LemMA 1.2. Let $u \in H_{r}^{1}\left(\mathbf{R}^{n}\right)$. Then

$$
|u(x)| \leqslant C_{n}|x|^{(1-n) / 2}\|u\| \quad \text { a.e., }
$$

where $C_{n}$ depends only on $n$, and the inclusion map

$$
H_{r}^{1}\left(\mathbf{R}^{n}\right) \hookrightarrow L_{r}^{p}\left(\mathbf{R}^{n}\right), \quad 2<p<2+4 /(n-2),
$$

is compact.

Proof. See Strauss [10].

LemmA 1.3. If $u_{0} \in H_{r}^{1}\left(\mathbf{R}^{n}\right)$ and $\int G\left(\left|u_{0}\right|\right) d x<\infty$ is a solution of (1.1), then $K\left(u_{0}\right)=0$. 
Proof. Let $u_{\beta}(x)=u_{0}(x / \beta)$. Then

$$
J\left(u_{\beta}\right)=\frac{1}{2} \int\left|\nabla u_{\beta}\right|^{2} d x+\int G\left(\left|u_{\beta}\right|\right) d x=\frac{\beta^{n-2}}{2} \int\left|\nabla u_{0}\right|^{2} d x+\beta^{n} \int G\left(\left|u_{0}\right|\right) d x .
$$

Since $u_{0}$ is a solution of $(1.1)$, then $\delta J\left(u_{0}\right)=0$, or $d\left(J\left(u_{\beta}\right)\right) /\left.d \beta\right|_{\beta=1}=0$. But

$$
\left.\frac{d\left(J\left(u_{\beta}\right)\right)}{d \beta}\right|_{\beta=1}=\frac{n-2}{2} \int\left|\nabla u_{0}\right|^{2} d x+n \int G\left(\left|u_{0}\right|\right) d x .
$$

Then $K\left(u_{0}\right)=0$.

LEMMA 1.4. $M$ is a $C^{1}$ hypersurface in $H_{r}^{1}\left(\mathbf{R}^{n}\right)$ bounded away from zero.

Proof. Since

$$
M=\left\{u: K(u)=\frac{n-2}{2} \int|\nabla u|^{2} d x+n \int G(|u|) d x=0, u \neq 0\right\}
$$

and $f=G^{\prime}$ is continuous, then $K(u)$ is a $C^{1}$ functional. Now consider $u_{0} \in H_{r}^{1}\left(\mathbf{R}^{n}\right)$ such that $\delta K\left(u_{0}\right)=0$

$$
-(n-2) \Delta u_{0}+n f\left(\left|u_{0}\right|\right) \arg u_{0}=0 .
$$

Then by Lemma 1.3 applied to equation (1.2) we have

$$
\frac{(n-2)^{2}}{2} \int\left|\nabla u_{0}\right|^{2} d x+n^{2} \int G\left(\left|u_{0}\right|\right) d x=0 .
$$

Therefore if $K\left(u_{0}\right)=0$ we have from (1.3)

$$
\frac{(n-2)^{2}}{2} \int\left|\nabla u_{0}\right|^{2} d x-\frac{n(n-2)}{2} \int\left|\nabla u_{0}\right|^{2} d x=0
$$

and this implies $u_{0}=0$. Hence for $u_{0} \in M, \delta K\left(u_{0}\right) \neq 0$ and $M$ is a $C^{1}$ hypersurface. Finally since $f^{\prime}(0)=1$ and $\lim f(|u|) /|u|^{\prime} \geqslant 0, l=1+4 /(n-2)$, we have $G(|u|) \geqslant$ $|u|^{2} / 4-C_{0}|u|^{1+1}$. Therefore

$$
K(u) \geqslant \frac{n-2}{2} \int|\nabla u|^{2} d x+\frac{1}{4} \int|u|^{2} d x-C_{0} \int|u|^{1+1} d x .
$$

From Sobolev embedding and $n>2$ we have $K(u) \geqslant\|u\|^{2} / 4-C_{0}\|u\|^{\alpha}$ where $\alpha>2$. For $0<\|u\|<\varepsilon, \varepsilon$ small, we have $K(u)>0$ and this implies that $M$ is bounded away from zero.

PROPOSITION 1.5 (EXISTENCE OF THE GROUND STATE). The minimization problem

$$
J_{0} \equiv \inf _{u \in M} J(u)=\inf \left\{\frac{1}{n} \int|\nabla u|^{2} d x, K(u)=0, u \neq 0\right\}
$$

which is equal to

$$
J_{0}=\inf \left\{\frac{1}{n} \int|\nabla u|^{2} d x, K(u) \leqslant 0, u \neq 0\right\}
$$

is attained at $u_{3} \in M\left(u_{e} \in H_{r}^{1}\left(\mathbf{R}^{n}\right)\right)$. 
Proof. First we establish the equivalence of the two minimizations. For any $v_{0} \in H_{r}^{1}\left(\mathbf{R}^{n}\right)$ such that $K\left(v_{0}\right)<0$ let $v_{\beta}(x)=v_{0}(x / \beta)$. Then

$$
K\left(v_{\beta}\right)=\frac{\beta^{n-2}(n-2)}{2} \int\left|\nabla v_{0}\right|^{2} d x+\beta^{n} n \int G\left(\left|v_{0}\right|\right) d x .
$$

Now for $\beta=1, K\left(v_{1}\right)=K\left(v_{0}\right)<0$ and for $\beta$ close to zero $K\left(v_{\beta}\right)>0$ since $n-2>0$. Therefore there is a $\beta_{0} \in(0,1)$ such that $K\left(v_{\beta_{0}}\right)=0$ (i.e. $\left.v_{\beta_{0}} \in M\right)$ ). But

$$
J\left(v_{\beta_{0}}\right)=\frac{1}{n} \int\left|\nabla v_{\beta_{0}}\right|^{2} d x=\frac{\beta_{0}^{n-2}}{n} \int\left|\nabla v_{0}\right|^{2} d x<\frac{1}{n} \int\left|\nabla v_{0}\right|^{2} d x
$$

since $\beta_{0} \in(0,1)$. Consequently the two minimizations are equivalent.

Let $u_{j} \in H_{r}^{1}\left(\mathbf{R}^{n}\right)$ be a minimizing sequence. Then $\int\left|\nabla u_{j}\right|^{2} d x$ is bounded. Since $\underline{\lim } f(|u|) /|u|^{l} \geqslant 0$, there is $C_{f}>0$ such that $G(|u|) \geqslant|u|^{2} / 4-C_{f}|u|^{l+1}$. Therefore

$$
\begin{aligned}
0 & \geqslant K\left(u_{j}\right)=\frac{n-2}{2} \int\left|\nabla u_{j}\right|^{2} d x+n \int G\left(\left|u_{j}\right|\right) d x \\
& \geqslant \frac{n-2}{2} \int\left|\nabla u_{j}\right|^{2} d x+\frac{n}{4} \int\left|u_{j}\right|^{2} d x-C_{f} \int\left|u_{j}\right|^{l+1} d x .
\end{aligned}
$$

By the Sobolev embedding $H_{r}^{1}\left(\mathbf{R}^{n}\right) \hookrightarrow L_{r}^{1+1}\left(\mathbf{R}^{n}\right)$ and the boundedness of $\int\left|\nabla u_{j}\right|^{2} d x$ we have $\left\|u_{j}\right\|$ is bounded. Therefore there is a subsequence, also denoted by $u_{j}$, such that

$$
u_{j} \stackrel{w}{\rightarrow} u_{e} \in H_{r}^{1}\left(\mathbf{R}^{n}\right)
$$

and

$$
u_{j} \rightarrow u_{e} \in L_{r}^{p}\left(\mathbf{R}^{n}\right), \quad 2<p<2+4 /(n-2) \quad \text { (by compactness). }
$$

Now by lower semicontinuity of weak limits and assumption (ii) we have

$$
\frac{1}{n} \int\left|\nabla u_{e}\right|^{2} d x \leqslant \underline{\lim } \frac{1}{n} \int\left|\nabla u_{j}\right|^{2} d x=J_{0}
$$

and $K\left(u_{e}\right) \leqslant \underline{\lim } K\left(u_{j}\right) \leqslant 0$. By the definition of $J_{0}$ the above inequalities are equalities and the weak convergence is strong.

THEOREM 1.6.

$$
-\Delta u+f(|u|) \arg u=0
$$

has a nontrivial (least energy) solution, namely $u_{e}$.

Proof. Since $u_{e}$ is a critical point of $J(u)$ restricted to $M$, by the Lagrange multiplier we have

$$
\delta J\left(u_{e}\right)+\eta \delta K\left(u_{e}\right)=0, \quad \eta \in \mathbf{R},
$$

or

$$
-\Delta u_{e}+f\left(\left|u_{e}\right|\right) \arg u_{e}+\eta\left(-(n-2) \Delta u_{e}+n f\left(\left|u_{e}\right|\right) \arg u_{e}\right)=0
$$




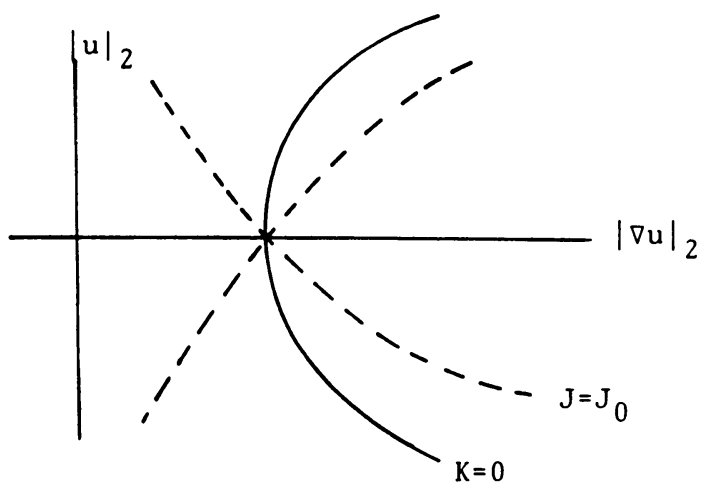

Figure 1

By Lemma 1.3 we have

$$
K\left(u_{e}\right)+\eta\left[\frac{(n-2)^{2}}{2} \int\left|\nabla u_{e}\right|^{2} d x+n^{2} \int G\left(\left|u_{e}\right|\right) d x\right]=0
$$

or

$$
K\left(u_{e}\right)+\eta\left[\frac{(n-2)^{2}}{2}-\frac{n(n-2)}{2} \int\left|\nabla u_{e}\right|^{2} d x+n K\left(u_{e}\right)\right]=0
$$

But $K\left(u_{e}\right)=0$ since $u_{e} \in M$. Hence $\eta \int\left|\nabla u_{e}\right|^{2} d x=0$ and this implies that $\eta=0$ and $u_{e}$ is a solution of (1.1).

REMARK. If we draw the picture in the space $H_{r}^{1}\left(\mathbf{R}^{n}\right)$ of $K(u)=0$ and $J(u)=J_{0}$ (which is not a manifold) we get formally Figure 1.

2. Instability of the ground state. Consider the Cauchy problem for equation (0.1)

$$
\begin{gathered}
u_{t t}-\Delta u+f(|u|) \arg u=0, \\
u(0)=u_{0} \in H_{r}^{1}\left(\mathbf{R}^{n}\right), \quad u_{t}(0)=u_{1} \in L_{r}^{2}\left(\mathbf{R}^{n}\right) .
\end{gathered}
$$

Definition 2.1. Let $X=\left\{u \in H_{r}^{1} ; \int G(|u|) d x<\infty\right\}$ and define the energy functional for equation $(0.1)$ as

$$
\begin{aligned}
& E(u, v)=\frac{1}{2} \int|v|^{2} d x+J(u), \quad u \in X, v \in L_{r}^{2}, \\
R_{1}= & \left\{u \in X, v \in L_{r}^{2}: E(u, v)<J_{0} \text { and } K(u)>0\right\} \cup\{(0,0)\} \\
= & \left\{u \in X, v \in L_{r}^{2}: E(u, v)<J_{0} \text { and } \frac{1}{n} \int|\nabla u|^{2} d x<J_{0}\right\}, \\
R_{2}= & \left\{u \in X, v \in L_{r}^{2}: E(u, v)<J_{0} \text { and } K(u)<0, u \neq 0\right\} \\
= & \left\{u \in X, v \in L_{r}^{2}: E(u, v)<J_{0} \text { and } \frac{1}{n} \int|\nabla u|^{2} d x>J_{0}\right\} .
\end{aligned}
$$

The Cauchy problem does not necessarily have strong solutions $u(\cdot) \in C\left([0, T), H^{1}\right)$ and $u_{t}(\cdot) \in C\left([0, T), L^{2}\right)$ for the type of nonlinearity we are considering. 
Definition. By a solution of $(0.1)$ on the time interval $[0, T)$ we mean a function $u(x, t)$ such that:

(1) $u\left(\right.$ resp. $u_{t}$ ) is weakly continuous in $t$ on $[0, T)$ with values in $H_{r}^{1}\left(\mathbf{R}^{n}\right)$ (resp. $\left.L_{r}^{2}\left(\mathbf{R}^{n}\right)\right)$.

(2) $E\left(u(t), u_{t}(t)\right) \leqslant E\left(u_{0}, u_{1}\right)$.

(3) $u$ satisfies $(0.1)$ in the sense of distributions.

If $u_{0} \in X, u_{1} \in L_{r}^{2}$ and $f$ satisfies (ii) of $\S 1$, then a solution exists on some time interval $[0, T)$. Moreover if $f$ satisfies the weak hypothesis (basically $|u| f(|u|) \geqslant$ $-C|u|^{2}$ for $u$ large), then the solutions exist globally in time [8]. Uniqueness is also not known for general $f[4,8]$, neither is regularity when $u_{0}$ and $u_{1}$ are smooth [8].

We multiply equation $(0.1)$ by $\phi_{m}(r, t) \bar{u}_{r}$, where

$$
\begin{aligned}
\phi_{m}(r, t)= & r H(m-r)+(\ln (m) r / \ln (r))(H(r-m)-H(r-(t+2 m))) \\
& +(\ln (m)(t+2 m) / \ln (t+2 m)) H(r-(t+2 m))
\end{aligned}
$$

and $H(s)=1, s \geqslant 0, H(s)=0, s<0, \ln$ is the natural $\log$ function, and take the real part

$$
\begin{aligned}
& \frac{d}{d t} \int \operatorname{Re} \phi_{m} \bar{u}_{r} u_{t} d x+\int_{r<m}\left(n\left|u_{t}\right|^{2} / 2-(n-2)|\nabla u|^{2} / 2-n G(|u|)\right) d x \\
& \quad+\int_{m<r<2 m+t}(\ln (m) / \ln (r))\left(n\left|u_{t}\right|^{2} / 2-(n-2)|\nabla u|^{2} / 2-n G(|u|)\right) d x \\
& \quad-\int_{m<r<2 m+t}\left(\ln (m) / \ln ^{2}(r)\right)\left(\left|u_{t}\right|^{2} / 2+|\nabla u|^{2} / 2-G(|u|)\right) d x \\
& \quad+\int_{2 m+t<r} \operatorname{Re} e_{1 m} \bar{u}_{r} u_{t}+e_{2 m}\left(\left|u_{t}\right|^{2}-|\nabla u|^{2}+2 G(|u|)\right) d x=0,
\end{aligned}
$$

where $\left|e_{i m}(r, t)\right|<C$ for $r>2 m+t$. Integrating with respect to $t$ we obtain

$$
\begin{aligned}
& \int \operatorname{Re} \phi_{m}(r, t) \bar{u}_{r} u_{t} d x-\int \operatorname{Re} \phi_{m}(r, 0) \bar{u}_{r}(0) u_{t}(0) d x \\
& =\int_{0}^{t} \int_{r<m}\left(-n\left|u_{t}\right|^{2} / 2+(n-2)|\nabla u|^{2} / 2+n G(|u|)\right) d x d s \\
& \quad+\int_{0}^{t} \int_{m<r<2 m+s}(\ln (m) / \ln (r))\left(-n\left|u_{t}\right|^{2} / 2+(n-2)|\nabla u|^{2} / 2+G(|u|)\right) d x d s \\
& \quad+\int_{0}^{t} \int_{m<r<2 m+s}\left(\ln (m) / \ln ^{2}(r)\right)\left(\left|u_{t}\right|^{2} / 2+|\nabla u|^{2} / 2-G(|u|)\right) d x d s \\
& \quad+\int_{0}^{t} \int_{r>2 m+s} \operatorname{Re}\left[e_{1 m} \bar{u}_{r} u_{t}+e_{2 m}\left(\left|u_{t}\right|^{2}-|\nabla u|^{2}+2 G(|u|)\right)\right] d x d s .
\end{aligned}
$$

Equation (2.2) is all that is needed to show instability of the ground state and in the appendix we will show that (2.2) holds for radial solutions as defined. 
LEMMA 2.2. $R_{1}$ and $R_{2}$ are invariant regions under the flow of $(0.1)$ for the solutions that satisfy the energy inequality.

Proof. We will show this by contradiction. Let $\left(u_{0}, u_{1}\right) \in R_{1}$ and assume there is a $t_{0}$ such that $\left(u\left(t_{0}\right), u_{t}\left(t_{0}\right)\right) \notin R_{1}$. By lower semicontinuity of $K(u(t))$ there is a minimal $t_{1}$ such that $\left(u\left(t_{1}\right), u_{t}\left(t_{1}\right)\right) \notin R_{1}$, i.e. $K\left(u\left(t_{1}\right)\right) \leqslant 0$ and $K(u(t))>0,0 \leqslant t$ $<t_{1}$. Now

$$
\begin{aligned}
& \frac{1}{n} \int\left|\nabla u\left(t_{1}\right)\right|^{2} d x \leqslant \lim _{\substack{t \rightarrow t_{1} \\
t<t_{1}}} \frac{1}{n} \int|\nabla u(t)|^{2} d x \\
& \leqslant \frac{\lim }{\substack{t \rightarrow t_{1} \\
t<t_{1}}}\left(\frac{1}{n} \int|\nabla u(t)|^{2} d x+\frac{1}{n} K(u(t))\right)
\end{aligned}
$$

and therefore

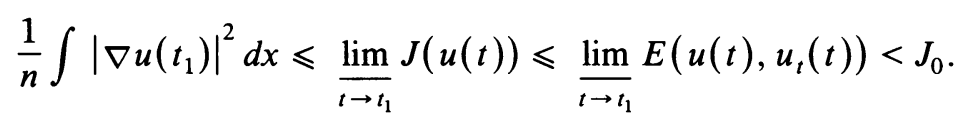

But we also have $K\left(u\left(t_{1}\right)\right) \leqslant 0$ and this contradicts the definition of $J_{0}$ as the inf of $(1 / n) \int|\nabla u|^{2} d x$ restricted to $K(u) \leqslant 0$ and $u \neq 0$. Therefore $R_{1}$ is an invariant region. Similarly we can show that $R_{2}$ is also invariant.

THEOREM 2.3. Consider solutions of (0.1) that are radially symmetric and satisfy the energy inequality with Cauchy data $\left(u_{0}, u_{1}\right) \in R_{2}$. Then either

(i) the solutions exist only locally in time $\left[0, T_{0}\right), T_{0}<\infty$, with $\left\|u\left(t_{n}\right)\right\| \rightarrow \infty$ as $t_{n} \rightarrow T_{0}$ for some sequence $\left(t_{n}\right)$, or

(ii) the solutions exist globally $[0, \infty)$ and $\exists$ a sequence $t_{n} \rightarrow \infty$ such that $\left\|u\left(t_{n}\right)\right\| \rightarrow$ $\infty$ as $t_{n} \rightarrow \infty$.

In either case the ground state is unstable.

Proof. Statement (i) is just a consequence of the local existence of the solution [8].

Now we show (ii) by contradiction. Assume that the solution remains bounded in $H_{r}^{1}\left(\mathbf{R}^{n}\right)$. Then there is an $\varepsilon>0$ such that $K(u(t))<-\varepsilon \forall t$ since otherwise we would have a sequence $\left(t_{k}\right)$ such that (since $R_{2}$ is invariant) $K\left(u\left(t_{k}\right)\right) \rightarrow 0$.

$$
\frac{1}{n} \int\left|\nabla u\left(t_{k}\right)\right|^{2} d x=J\left(u\left(t_{k}\right)\right)-\frac{1}{n} K\left(u\left(t_{k}\right)\right)<E\left(u_{0}, u_{1}\right)-\frac{1}{n} K\left(u\left(t_{k}\right)\right)
$$

and if we extract a weakly convergent subsequence $u\left(t_{k}\right) \stackrel{w}{\rightarrow} u^{*} \in H_{r}^{1}\left(\mathbf{R}^{n}\right), u\left(t_{k}\right) \rightarrow u^{*}$ $\in L_{r}^{p}, 2<p<2+4 /(n-2)$, we get

$$
\frac{1}{n} \int\left|\nabla u^{*}\right|^{2} d x \leqslant E\left(u_{0}, u_{1}\right)<J_{0}
$$

(since $\left.\left(u_{0}, u_{1}\right) \in R_{2}\right)$ and $K\left(u^{*}\right) \leqslant 0$. But this contradicts the definition of $J_{0}$. Therefore such an $\varepsilon$ exists. 
Now from Lemma 1.2 we have $|u(r, t)| \leqslant C_{n}\|u(t)\| r^{(1-n) / 2}<C_{n} C r^{(1-n) / 2}$ and this implies

$$
G(|u(r, t)|)=\int_{0}^{|u(r, t)|} f(s) d s \geqslant 0 \text { for } r \text { large. }
$$

Now the right-hand side of equation (2.2) can be bounded above as follows:

$$
\begin{gathered}
\int_{m<r<2 m+t}\left(n \ln (m) / \ln (r)-\ln (m) /(\ln (r))^{2}\right) G(|u|) d x<n \int_{m<r} G(|u|) d x, \\
\int_{m<r<2 m+t}\left((n-2) \ln (m) /(2 \ln (r))+\ln (m) / 2(\ln (r))^{2}\right)|\nabla u|^{2} d x \\
<\frac{n-2}{2} \int_{m<r}|\nabla u|^{2} d x+\frac{1}{2 \ln (m)} \int_{m<r}|\nabla u|^{2} d x \\
\int_{r>2 m+t}\left(\left|\bar{u}_{r} u_{t}\right|+\left|u_{t}\right|^{2}+|\nabla u|^{2}+|G(|u|)|\right) d x \\
\leqslant C_{0}\left(\int_{2 m<r}\left|\nabla u_{0}\right|^{2}+\left|u_{1}\right|^{2}+G\left(\left|u_{0}\right|\right) d x\right)
\end{gathered}
$$

since radial solutions are strong outside a light cone containing the origin and therefore can be estimated by the initial data at the base. Consequently (2.2) will be bounded above by

$$
\begin{aligned}
\int \operatorname{Re} \phi_{m}(r, t) & \bar{u}_{r}(r, t) u_{t}(r, t) d x-\int \operatorname{Re} \phi_{m}(r, 0) \bar{u}_{r} u_{1} d x \\
\leqslant & \int_{0}^{t}\left(K(u(s))+\frac{1}{2 \ln (m)} \int_{m<r}|\nabla u|^{2} d x\right. \\
& \left.+C_{1} \int_{2 m<r}\left(\left|\nabla u_{0}\right|^{2}+\left|u_{0}\right|^{2}+\left|u_{1}\right|^{2}+G\left(\left|u_{0}\right|\right)\right) d x\right) d s .
\end{aligned}
$$

Choose $m$ large enough so that

$$
\frac{1}{2 \ln (m)} \int|\nabla u|^{2} d x<\frac{\varepsilon}{4}, \quad C_{1} \int_{2 m<r}\left(\left|\nabla u_{0}\right|^{2}+\left|u_{0}\right|^{2}+G\left(\left|u_{0}\right|\right)\right) d x<\frac{\varepsilon}{4} .
$$

Since $K(u(t))<-\varepsilon$ equation (2.3) becomes

$$
\int \operatorname{Re} \phi_{m} \bar{u}_{r} u_{t} d x-\int \operatorname{Re} \phi_{m}(r, 0) \bar{u}_{0 r} u_{1} d x \leqslant-\varepsilon t / 2
$$

but $\left|\phi_{m}(r, t)\right| \leqslant C t / \ln (t)$ for $t$ large. Then (2.4) implies that

$$
|\nabla u(t)|_{2}\left|u_{t}(t)\right|_{2}>C(\varepsilon) \ln (t) \text { for } t \text { large. }
$$

This contradicts the assumption that $u(t)$ is bounded in $H_{r}^{1}\left(\mathbf{R}^{n}\right)$. Therefore there is a sequence $\left(t_{n}\right)$ such that $\left\|u\left(t_{n}\right)\right\| \rightarrow \infty$.

Finally to show that this implies instability we note that for $u_{0}(x)=u_{e}(x / \lambda)$, $\lambda>1, u_{1}(x)=0$, we have $\left(u_{0}, u_{1}\right) \in R_{2}$ and is close to the ground state. 
REMARK. It is worthwhile noticing that if the initial data are in $R_{1}$ then there is a global solution with this initial data. To see this by the energy inequality we have

$$
\frac{1}{2} \int\left|u_{t}\right|^{2} d x+\frac{1}{n} \int|\nabla u|^{2} d x+\frac{1}{n} K(u)<J_{0}
$$

and since $R_{1}$ is invariant, then $K(u(t))>0 \forall t$. Therefore

$$
\frac{1}{2} \int\left|u_{t}\right|^{2} d x+\frac{1}{n} \int|\nabla u|^{2} d x \leqslant J_{0} \text { and } \int G(|u(t)|) d x
$$

are bounded and this implies that we can find a global solution.

Appendix. To show that (2.2) holds for weak radial solutions we will follow the method of Strauss [11] to prove that the solutions are continuous in $t$ off the origin.

LEMMA A. For every $\delta>0$ the weak radial solutions $u$ are

$$
u \in C\left([0, T), H_{r}^{1}\left(\mathbf{R}^{n} \backslash B_{\delta}\right)\right), \quad u_{t} \in C\left([0, T), L_{r}^{2}\left(\mathbf{R}^{n} \backslash B_{\delta}\right)\right)
$$

for all $T$ where the solutions exist $\left[B_{\delta}=\{r, r<\delta\}\right]$.

Proof. Let $\rho_{k}$ be an approximate identity and $u_{k}(t)=\rho_{k} * u(t)$. Then $u_{k}(t) \rightarrow$ $u(t)$ in $H_{r}^{1}\left(\mathbf{R}^{n}\right)$. Let $\chi_{\delta}(r)$ be a smooth function such that $\chi_{\delta}(r)=0, r<\delta / 2$, and $\chi_{\delta}(r)=1, r>\delta$.

Convoluting equation $(0.1)$ with $\rho_{k}$ we obtain

$$
u_{k t t}-\Delta u_{k}+\rho_{k} * f(|u|) \arg u=0 .
$$

Multiply (A.1) by $\chi_{\delta} \bar{u}_{k t}$ to get

$$
\begin{aligned}
\frac{1}{2} \frac{d}{d t} & \int\left(\left|u_{k t}\right|^{2}+\left|\nabla u_{k}\right|^{2}\right) \chi_{\delta} d x \\
& \quad-\int \operatorname{Re}\left(\chi_{\delta}\left(\rho_{k} * f(|u|)\right) \bar{u}_{k t}+\chi_{\delta r} u_{k r} \bar{u}_{k t}\right) d x=0 .
\end{aligned}
$$

Now since $u \in L^{\infty}\left([0, T), H^{1}\left(\mathbf{R}^{n}\right)\right)$ and is radially symmetric, then by Lemma 1.1 $|u(r, t)|<c$ for $r>\delta$ and this implies that $\chi_{\delta}\left(\rho_{k} * f(|u|)\right) \rightarrow \chi_{\delta} f(|u|) \in$ $L^{\infty}\left([0, T), L_{r}^{2}\left(\mathbf{R}^{n}\right)\right)$ and passing to the limit as $k \rightarrow \infty$ in (A.2) we get the desired result.

LEMMA B. Equation (2.2) holds for weak radial solutions.

Proof. As before we work with equation (A.1). Multiply (A.1) by $\psi_{\delta, m}(r, t) \bar{u}_{k r}(r, t)$ where

$$
\psi_{\delta, m}(r, t)= \begin{cases}0, & r<\delta, \\ (r-\delta) m /(m-\delta), & \delta<r<m, \\ \phi_{m}(r, t), & r>m,\end{cases}
$$


$\phi_{m}(r, t)$ is defined in $\S 2$. Then we have

(A.3)

$$
\begin{aligned}
& \frac{d}{d t} \int \operatorname{Re} \psi_{\delta, m} \bar{u}_{k r} u_{k t} d x-\int \operatorname{Re} \psi_{\delta, m} \bar{u}_{k r} u_{k t} d x \\
& \quad+\int\left(r^{n-1} \psi_{\delta, m}\right)_{r}\left|u_{k t}\right|^{2} / 2 r^{n-1} d x \\
& \quad-\int\left((n-1) \psi_{\delta, m} / r-\left(r^{n-1} \psi_{\delta, m}\right)_{r} / 2 r^{n-1}\right)\left|\nabla u_{k}\right|^{2} d x \\
& \quad+\int \operatorname{Re} \rho_{k} * f(|u|) \arg u \psi_{\delta, m} \bar{u}_{k r} d x=0 .
\end{aligned}
$$

As before we integrate with respect to $t$ and take the limit as $k \rightarrow \infty$ where each integral term converges to its proper value:

$$
\begin{aligned}
\int \rho_{k} * f(|u|) \arg u \psi_{\delta, m} \bar{u}_{k r} d x \rightarrow \int f(|u|) \arg u \psi_{\delta, m} \bar{u}_{r} d x \\
=-n \int_{\delta<r<m} m /(m-\delta) G(|u|) d x+\text { terms independent of } \delta .
\end{aligned}
$$

Let $\delta \rightarrow 0$. Then from the above equation and (A.3) we obtain equation (2.2).

\section{REFERENCES}

1. D. L. T. Anderson, J. Math. Phys. 12 (1971), 945-952.

2. H. Berestycki and T. Cazenave (to appear).

3. G. H. Derrick, Comments on nonlinear wave equations as models for elementary particles, J. Math. Phys. 5 (1964), 1252-1254.

4. R. Glassey and M. Tsutsumi, On uniqueness of weak solutions to semilinear wave equations, Comm. Partial Differential Equations 7 (1982), 153-195.

5. Clayton Keller (to appear).

6. L. E. Payne and D. H. Sattinger, Saddle points and instability of nonlinear hyperbolic equations, Israel J. Math. 22 (1975), 272-303.

7. J. M. Shatah, Stable standing waves of nonlinear Klein-Gordon equations, Comm. Math. Phys. 91 (1983), 313-327.

8. W. A. Strauss, An. Acad. Brasil. Ciênc. 42 (1970), 645-651.

9. N__ Nonlinear invariant waves equations, Lecture Notes in Phys., vol. 73, Springer, Berlin, Heidelberg, and New York, 1978, pp. 197-249.

10. __ Existence of solitary waves in higher dimensions, Comm. Math. Phys. 55 (1977), 149-162.

11. On continuity of functions with values in various Banach spaces, Pacific J. Math. 19 (1966), $543-551$.

12. H. Berestycki and P. L. Lions, Existence d'onde solitaires dans les problèmes non-linéaires du type Klein-Gordon, Arch. Rational Mech. Anal. 82 (1983), 316-338.

13. T. Cazenave, Uniform estimates of solutions to nonlinear Klein-Gordon equations, Univ. Pierre et Marie Curie Lab. d'Analyse Numérique, preprint.

Division of Applied Mathematics, Brown University, Providence, Rhode Island 02912

Current address: Courant Institute of Mathematical Sciences, 251 Mercer Street, New York, New York 10012 